\title{
Mappare il virus \\ Strategie e pratiche di visualizzazione dei dati \\ legate al fenomeno COVID-19
}

Valentina Manchia

Politecnico di Milano, Dipartimento di Design; CROSS

valentina.manchia@polimi.it

\begin{abstract}
Never as in these months of global pandemic emergency there has been a real explosion of data, numbers, statistics and views in an attempt to make the invisible visible: the COVID-19 virus.

This essay proposes to explore, with the tools of semiotics, the different communicative and rhetorical strategies implemented by some visual artifacts to try to investigate the phenomenon through the visualization of data.
\end{abstract}

\section{Keywords}

Semiotics; Visual semiotics; Data visualization; Data journalism; COVID-19

\section{Sommario/Contents}

1. Fare guerra all'invisibile rendendolo visibile. Una premessa di metodo

2. I big data del virus tra comunicazione e visualizzazione classica

3. La narrazione dell'epidemia e altre forme di visualizzazione

3.1. Ripartire da nuovi dati. Dai big data ai thick data

3.2. Guardare ai dati con altri occhi. L'approccio narrativo ai big data Bibliografia 
Valentina Manchia | Mappare il virus

\section{Fare guerra all'invisibile rendendolo visibile. Una premessa di metodo}

In un recente articolo Frédérique Aït-Touati, storica della scienza, invita a rileggere, alla luce di questi tempi, Les Microbes: guerre et paix di Bruno Latour, su Pasteur e sulla "pastorizzazione" della scienza moderna.

La storia di Pasteur e della sua capitale scoperta, l'esistenza dei microbi, che segna l'inizio a tutti gli effetti della scienza come la conosciamo, è esemplare anche e soprattutto dal punto di vista della sociologia della scienza: Les microbes racconta infatti l'entrata, sulla scena non solo scientifica ma anche economica, politica e sociale, di un nuovo attore: un attore non-umano, potenzialmente aggressivo, che occorre comprendere e gestire, prima ancora che combattere.

Se la metafora scenica è estremamente adeguata dal punto di vista narrativo, segnando l'ingresso, nella storia, di un Anti-soggetto dotato di esigenze e volontà opposte al Soggetto per eccellenza, l'uomo, è interessante notare come, a livello discorsivo (nello specifico, nella gran quantità di discorsi, documenti e materiali analizzati da Latour per la sua ricerca) l'introduzione di questa nuova entità non-umana nella società degli umani sia accompagnata da un'importante trasformazione, quella da entità invisibile a entità visibile, per la mediazione delle procedure scientifiche. ${ }^{1}$

Nello specifico, il cambio radicale di regime di visibilità avviene nel laboratorio di Pasteur, accuratamente studiato (secondo protocolli radicalmente diversi da quelli in voga all'epoca) per regolamentare, tracciare e documentare il passaggio da invisibile a visibile e allo stesso tempo per rendere evidente e comunicabile tale scoperta: il laboratorio è pertanto un vero e proprio «théâtre de la preuve», il luogo in cui la scoperta scientifica diventa evidenza scientifica a tutti gli effetti, ovvero anello di congiunzione tra i protocolli di laboratorio e la loro efficacia dimostrativa e l'apparizione, agli occhi della comunità scientifica e sociale, della scoperta come evidenza a tutti gli effetti.

Nel passaggio riportato qui in basso, sono le procedure di produzione dei dati e i dati stessi, come risultato delle indagini di laboratorio, a tracciare in modo visibile l'esistenza dell'invisibile:

C'est surtout un lieu où les microbes sont pour ainsi dire à découvert, et donc enfin visibles : « à la ferme, il y a des veaux, des vaches, des couvées, Perrette et son pot au lait et les saules le long de la mare. Difficile de repérer Rosette malade ou de la comparer. Difficile d'y voir quoi que ce soit si c'est un microbe qu'on cherche. Au labo, les chercheurs ont la colonie $\mathrm{n}^{\circ} 5,7,8$, avec les colonies témoins $\mathrm{n}^{\circ} 12,13,15$. Une feuille à double colonne avec des croix et des points. C'est tout. Il suffit de savoir lire. (Latour 1984: 93)

1 «La grande surprise du pastorisme est de faire apparaitre sur la scène du monde des milliards d'autres acteurs qui agissent, poursuivent des buts qui nous sont inconnus et se servent de nous pour prospérer. La science rend visible des acteurs jusque-là restés invisibles, qui sont non humains, et néanmoins agissent» (Aït-Touati 2020). 
Valentina Manchia | Mappare il virus

A leggere più da vicino queste righe, Latour è molto chiaro a proposito della forza euristica del laboratorio. In natura (qui la fattoria abitata da Perrette e dai suoi animali, presa in prestito dall'immaginario fiabesco di Perrault) tutto è nitido e ricco di dettagli, come in una pittoresca veduta, ma è impossibile rilevare la presenza dei microbi, abitanti invisibili di una dimensione che sfugge allo sguardo. È solo nel laboratorio, invece, luogo non di natura ma eminentemente culturale (e costruito, come è stato mostrato nell'ambito dei laboratory studies e degli Science \& Tecnology Studies), ${ }^{2}$ che appaiono i microbi, grazie all'intervento di strumenti e tecnologie che di fatto potenziano lo sguardo consegnando allo scienziato un nuovo tipo di vedere (esatto e misurabile) che non è quello dell'uomo comune. Lo sguardo potenziato dello scienziato agisce per settori, dividendo in campioni di colture e comparandoli tra loro: in ultimo, estrae dati, allineandoli in tabelle a doppia entrata.

È da dietro quei dati che emergono i microbi, che diventano visibili non solo su un vetrino di microscopio ma perché diventano numerabili e quantificabili, ed è grazie a quei dati che può avere inizio la guerra contro di loro.

Nella rilettura della teoria della costruzione dell'oggetto scientifico in Latour proposta da Françoise Bastide, biologa e semiologa, vicina a Latour ma allieva di Greimas, appare però chiaro come la fase di acquisizione ed elaborazione dei dati sia solo una fase intermedia: tra i dati e la loro comunicazione, tra il lavoro in laboratorio (qui «la colonie $\mathrm{n}^{\circ} 5,7,8$, avec les colonies témoins $\mathrm{n}^{\circ} 12,13,15$ ») e la comunicazione dei risultati scientifici («Une feuille à double colonne avec des croix et des points») c'è un ulteriore passaggio, un processo che Bastide non esita a definire traduttivo, quello dell'elaborazione di strategie per la messa in discorso di quei primi dati in una nuova forma:

Di norma non si pubblicano i risultati "grezzi", così come escono dalle apparecchiature di registrazione. Si fanno delle medie, si calcolano le varianti, si costruiscono rappresentazioni grafiche, si selezionano esperimenti "rappresentativi", e soprattutto non si pubblicano solamente i risultati. In effetti, oltre a una descrizione dei risultati, un articolo contiene un commento (discussione) e i differenti elementi adatti a inserire i risultati in un sistema destinato a metterli in valore, compreso il problema che ci si era proposti di risolvere (introduzione), la descrizione del dispositivo posto in opera (metodi e materiali), e una discussione sulla portata dei risultati ottenuti in una problematica più generale (conclusione). (Bastide 1985a: 223, tr. it.)

Detto questo, cosa accade se, seguendo Bastide che invita a considerare anche le pratiche di visualizzazione dei dati come vere e proprie strategie di messa in discorso, ${ }^{3}$ proviamo a spostare lo sguardo dai microbi di Pasteur e di Latour alle dinamiche e alle strategie di visualizzazione relative al nuovo attore patogeno apparso sulla scena, il virus COVID-19?

2 Cfr. in particolare Latour (1987) e (1996), ma anche Latour, Woolgar (1979). A proposito dei punti di contatto tra l'approccio di Latour e la semiotica, anche per il tramite di Françoise Bastide, cfr. anche Latour (2011), Latour, Woolgar (1979) e Latour, Fabbri (1977).

3 A questo proposito cfr. anche Fabbri, in Bastide (2001), sul testo scientifico come «manifestazione sincretica di un'attività tracciante» (Fabbri 2001: 14). 


\section{Ocula \\ Occhio semiotico sui media | Semiotic eye on media}

Valentina Manchia | Mappare il virus

Anche in questo recente (e purtroppo ancora attuale) caso ci troviamo a confronto con un soggetto (un Anti-soggetto) invisibile, e sulle strategie di rappresentazione adottate per quantificarlo, misurarlo, maneggiarlo.

Può essere dunque interessante, a nostro parere, provare a spostare l'attenzione su alcuni diversi esempi di visualizzazioni di dati e informazioni prodotte a partire dall'inizio dell'epidemia (gennaio-febbraio 2020) a oggi e ad analizzare le strategie adottate per cercare di rendere visibile il virus invisibile. Il tentativo sarà, parallelamente, di riportare alla luce le tracce della messa in discorso di tali dati e informazioni.

\section{I big data del virus tra comunicazione e visualizzazione clas- sica}

Sin dai primi accenni di diffusione di un virus tanto pericoloso quanto sconosciuto - a Wuhan, nella provincia cinese di Hubei, fin da dicembre 2019, e da gennaio 2020 anche in Italia, Giappone, Corea del Sud e Iran e da lì nel resto del mondo - le informazioni fornite dalla stampa mondiale in merito all'apparire del fenomeno sono state essenzialmente quantitative: numero di morti, numero di contagiati, numero di contagiati sottoposti a ospedalizzazione. Vista la gravità del fenomeno, i dati statistici sono stati da subito raccolti in modo capillare e puntuale in tutto il mondo (da governi, istituti di sanità e istituti di ricerca) e diffusi in modo altrettanto esaustivo.

In Italia, per esempio, a partire dal 31 gennaio 2020, con la dichiarazione dello stato di emergenza da parte del Consiglio dei Ministri per il rischio sanitario connesso all'infezione da Coronavirus, è stato diramato tutti i giorni, per due mesi, alle $18 \mathrm{~h} 30$, un bollettino da parte della Protezione Civile con la presentazione e il commento delle cifre del giorno riguardanti il virus (numero di guariti, deceduti, in terapia intensiva) e valutazioni in merito all'evoluzione del fenomeno rispetto ai giorni precedenti.

I bollettini della protezione civile, nel bel mezzo dell'emergenza nazionale italiana culminata nel lockdown, dal 9 marzo al 20 maggio 2020, hanno finito per diventare un vero e proprio appuntamento mediatico per ragionare collettivamente sulla situazione generale: seguiti e commentati su YouTube e sui social, rilanciati dai giornali e dalle televisioni, i trenta minuti del bollettino sono però stati più spesso dedicati alla lettura in diretta di alcune delle cifre più rilevanti, scelte tra i dati disponibili, e non a una presentazione sinottica dei dati in forma visiva, che avrebbe consentito, come vedremo meglio poco oltre, di comparare e confrontare meglio le informazioni (fig. 1).

L'esposizione orale dei dati da parte del capo della Protezione Civile si basa infatti sulla tabella ufficiale giornaliera, fornita ai giornalisti e agli organi di stampa (e alla quale spesso si fa riferimento anche durante il bollettino televisivo, proprio per ancorare il discorso a una descrizione referenziale e oggettiva della situazione), con i dati relativi al quadro italiano nella sua globalità (fig. 2). ${ }^{4}$

4 Del ruolo dei numeri nella costituzione dell'effetto di reale del racconto della pandemia ha detto anche Paolo Fabbri: «Il nudo numero, come diceva benissimo Bar- 


\section{Ocula ${ }^{\text {Flux_saggi }}$ \\ Occhio semiotico sui media | Semiotic eye on media}

Valentina Manchia | Mappare il virus

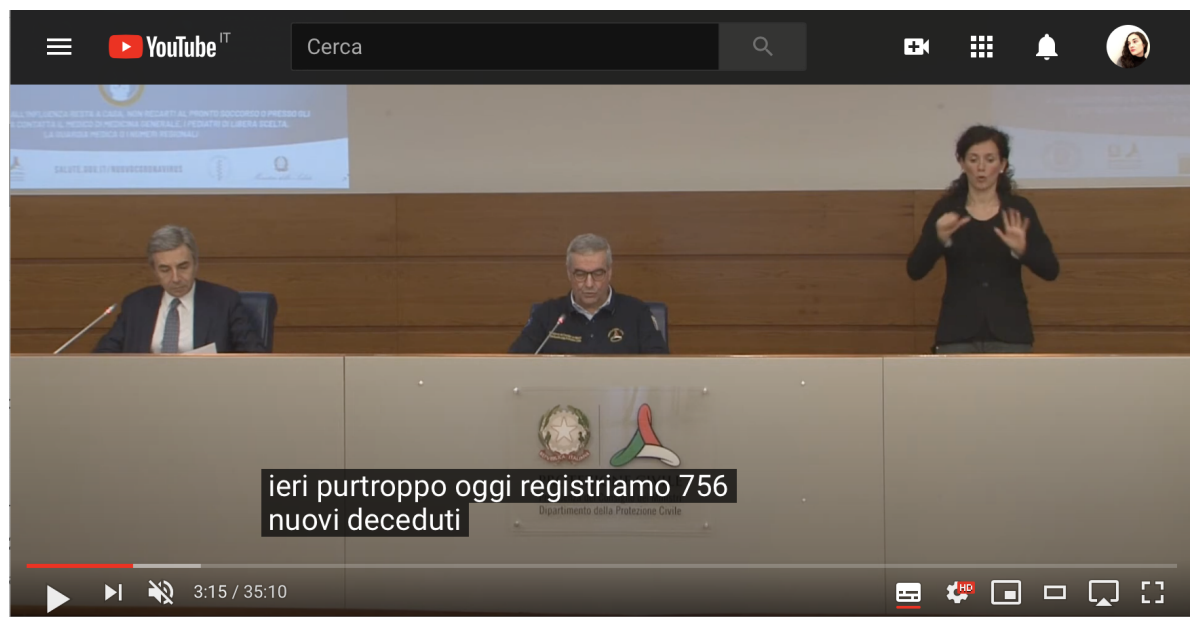

Riproduzione della chat dal vivo disattivata per questo video.

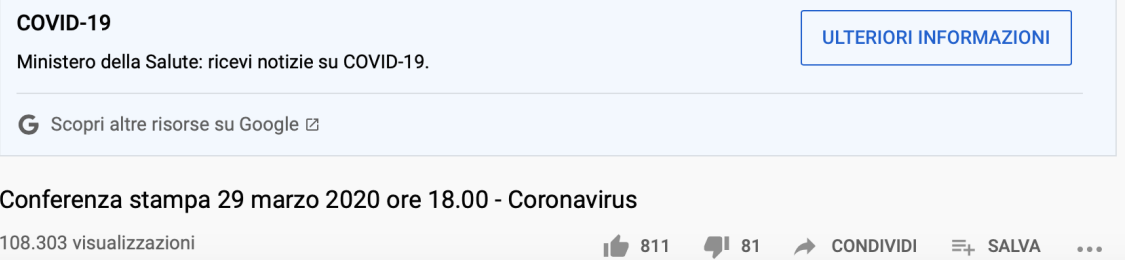

108.303 visualizzazioni

It $811 \rightarrow$ CONDIVIDI $81 \rightarrow+$ SALVA

Figura 1. Screenshot dalla conferenza stampa della Protezione Civile, 29 marzo 2020.

Fine comunicativo principale dei bollettini, in effetti, più che informare puntualmente sulla situazione, era quello di fornire ai destinatari del discorso, i cittadini, specifiche competenze (nel senso semiotico del termine) per affrontare al meglio la situazione attraverso le pratiche di contenimento del virus (igiene, distanziamento sociale, rispetto delle restrizioni, su cui ogni bollettino televisivo puntualmente ha insistito). L'obiettivo, infatti, è ottenere cittadini che possano ma soprattutto vogliano rispettare le regole di prevenzione e di restrizione, venendo a conoscenza della gravità della situazione generale.

In questo senso la modalità di comunicazione - vocale, ma quasi oracolare - dei dati numerici è funzionale all'obiettivo primario: quello di cementare comportamenti corretti e consapevoli, non soltanto attraverso un dover fare ma anche attraverso un poter e un voler fare, in piena adesione con l'andamento dell'emergenza. ${ }^{5}$

thes, è l'equivalente della pendola Luigi XIV quando la marchesa esce alle cinque. Perché, in un racconto, la pendola Luigi XIV e perché suona alle cinque? Poteva essere una qualunque pendola se si trattava di suonare a quell'ora; ma se tu dici, in un racconto, "pendola e Luigi XIV" rendi più verosimile l'evento, lo rendi "reale". Gli scienziati e in particolare gli addetti alla comunicazione scientifica non conoscono le teorie letterarie e della narrazione, forse anche noi studiosi delle scienze umane cominciamo a dimenticarcele» (Montanari, a cura di, 2020: 7):

5 Del bollettino della Protezione Civile come una sorta di rito collettivo e di evento mediatico parla, per esempio, Bonini (2020). 


\section{Dcula \\ Flux_Saggi \\ Occhio semiotico sui media | Semiotic eye on media}

Valentina Manchia | Mappare il virus

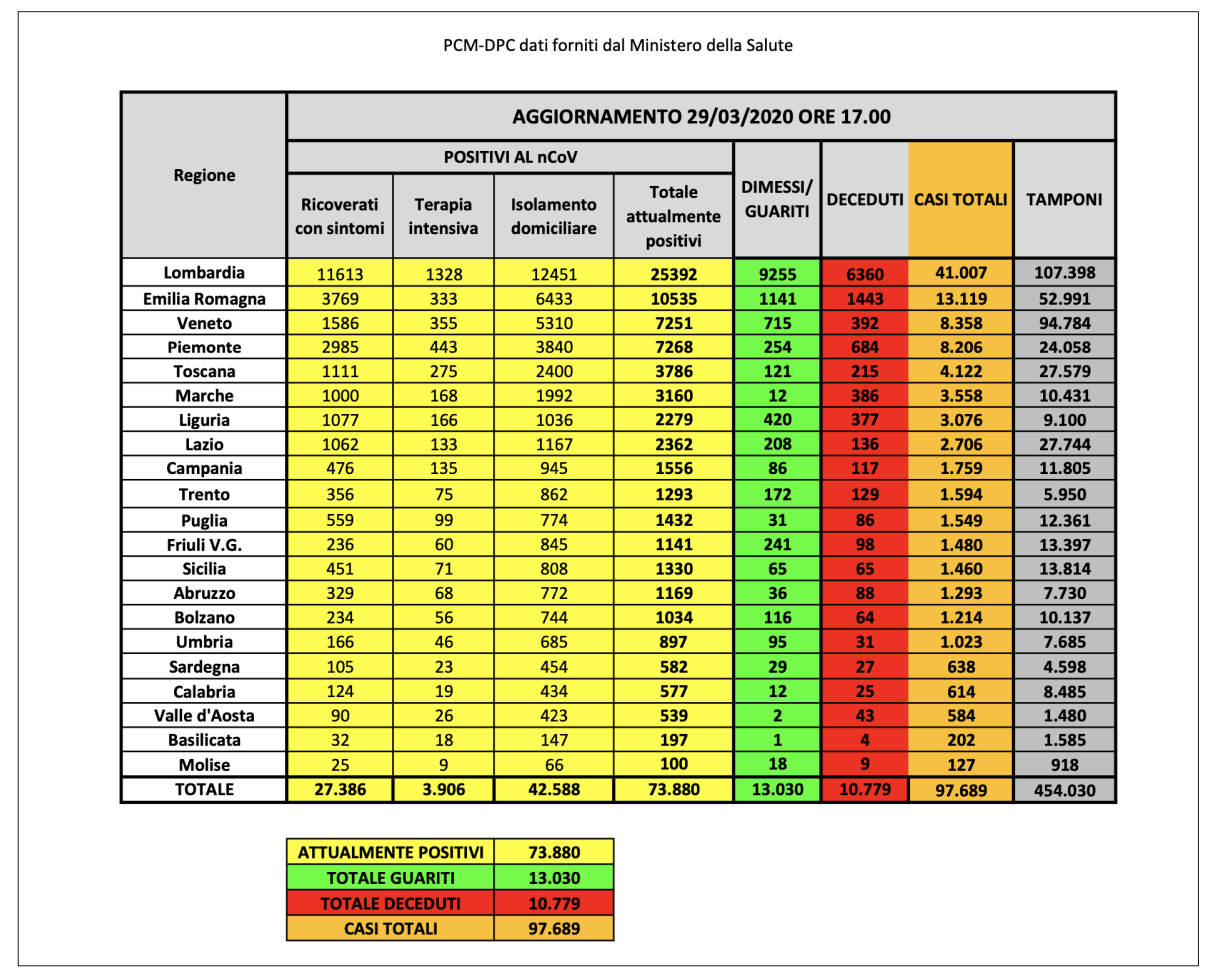

Figura 2. Tabella giornaliera della Protezione Civile, 29 marzo 2020.

Immediatamente a seguito della diffusione del bollettino giornaliero della Protezione Civile, nel caso italiano, anche gli organi di stampa sono naturalmente legittimati a fare appello agli stessi dati e a disporne anche in altro modo. Oltre a renderli oggetto di articoli e di approfondimenti, pertanto, giornali e mass media spesso hanno presentato e presentano i dati in forma di lista o nella stessa forma tabellare fornita dalla Protezione Civile, spesso con poche o nessuna variazione.

Dal punto di vista della restituzione dei dati, la forma tabellare consente al destinatario dell'informazione un tipo di acquisizione diversa dal semplice ascolto della comunicazione orale. Oltre alla maggiore maneggevolezza, per così dire, della forma scritta rispetto alla comunicazione orale (è notorio che la nascita della scrittura sia collegata allo sfruttamento di un supporto per l'iscrizione di informazioni complesse), ${ }^{6}$ la forma tabellare consente infatti di poter operare confronti e comparazioni tra i dati in modo molto più immediato della semplice restituzione scritta. ${ }^{7}$

Come si è ampiamente potuto notare nel corso di questi mesi di costante copertura mediatica della pandemia, sono state però le visualizzazioni dei dati relativi al virus in forma visiva ad aver avuto uno spazio ancora maggiore,

6 Ci riferiamo qui, in particolare, ai capitali Ong (1982) e Goody (1977). Di quest'ultimo, in particolare, ricordiamo l'analisi sulla struttura e sul funzionamento di un oggetto scritturale e contemporaneamente visivo come la lista.

7 Chi lavora sui dati e sulla loro restituzione insiste sulla necessità non soltanto di presentare i dati, ma anche di organizzarli in una forma coerente e di consentire comparazioni tra serie e correlazioni tra valori. Cfr. per esempio Cairo (2013). 


\section{Ocula ${ }^{\text {Flux_saggi }}$ \\ Occhio semiotico sui media | Semiotic eye on media}

Valentina Manchia | Mappare il virus

proprio per il maggior impatto che sono in grado di creare su una pagina o su un sito web, rendendo immediatamente visibile - e non solo maggiormente comprensibile - il fenomeno.

Bastide (1985b), in un altro dei suoi già citati saggi sul discorso scientifico, descrive bene lo specifico modo in cui tabelle e grafici rendono visibili i dati:

Esistono due forme di illustrazione che utilizzano nello stesso tempo i vantaggi del numero e la possibilità di sfruttare contemporaneamente almeno due dimensioni dello spazio. Sono le tabelle e i grafici che si dispongono entrambi sulle due dimensioni piane e rettangolari del foglio di carta, e facili da confrontare come le fotografie. (Bastide 1985b: 196, tr. it.)

A ben guardare, infatti, come evidenzia Bastide, anche le tabelle possono essere considerate una forma di visualizzazione dei dati: tuttavia, se una tabella "illustra" i dati rispetto alle possibili comparazioni tra tutti gli elementi che sceglie di porre in correlazione, un grafico degli stessi dati non offrirà la stessa possibilità di confronto e di lettura dei dati offerti in forma tabellare, perché pur presupponendo la stessa base di dati ne propone un'unica forma visiva:

L'uso di un grafico presuppone che sia stato effettuato un calcolo preliminare, in quanto costituisce la presentazione in altra forma di una tabella di cifre. (Bastide 1985b: 197, tr. it.; corsivo nostro)

E ancora, sempre continuando a leggere Bastide:

Quali sono i vantaggi rispettivi della tabella e del grafico? Sembra che sia un problema di leggibilità: il grafico si può interpretare con un unico colpo d'occhio, mentre la tabella richiede che si confrontino alcune cifre, che si facciano mentalmente sottrazioni e addizioni, moltiplicazioni e divisioni. Il grafico probabilmente è più "convincente", in quanto fa risparmiare tempo e attenzione al lettore. [...] In più, il grafico permette di rappresentare "la quarta dimensione", quella del tempo, traducendola come dimensione spaziale. (Bastide 1985b: 197, tr. it.)

Se dunque l'adottare una forma tabellare per la comunicazione dei dati si riconduce, in modo più o meno esplicito, al voler restituire al destinatario, insieme alla molteplicità di dimensioni che la tabella comprende, diverse possibilità di lettura dei dati, la scelta di una forma grafica (o infografica, come vedremo anche più avanti, per usare un termine anche troppo usato attualmente) presuppone l'adozione di un percorso interpretativo specifico di quei dati, e dunque la costruzione di un discorso orientato a partire da uno specifico punto di vista. Potremmo anche aggiungere che alla grande molteplicità di percorsi potenziali resi possibili dalla tabella si contrappone l'attualizzazione di uno specifico percorso di lettura attraverso la visualizzazione grafica - che pertanto risulta uno strumento più immediato, agli occhi del lettore, ma al contempo non fa che attualizzare uno specifico percorso di lettura.

Proprio per questo motivo, per la maggiore immediatezza che naturalmente tendiamo ad associare alla rappresentazione grafica dei dati rispetto 
Valentina Manchia | Mappare il virus

alla loro forma tabellare, unita alla necessità di trovare una forma, visibile e comunicabile, corrispondente alla gravità del fenomeno, si sono moltiplicati, sin dai primi mesi del 2020, gli esempi di data visualization applicati ai dati del COVID-19. ${ }^{8} \mathrm{E}$, sin da subito, il COVID-19 ha trovato nella curva dell'incremento dei contagi, nelle visualizzazioni cartesiane standard, il suo volto più riconoscibile, insieme all'invito ad "abbassare la curva". ${ }^{9}$ Poche altre volte la visualizzazione dei dati è diventata così pervasiva.

Un esempio tra i più noti, continuamente citato e riproposto da giornali e telegiornali, sul quale vale la pena di soffermarsi proprio per la sua centralità a livello mediatico, è senza dubbio la visualizzazione dei dati in forma di mappa di dati globale messa a punto dalla mappa della Johns Hopkins University, a opera del Center for Systems Science and Engineering, sin dai primi giorni dell'epidemia a Wuhan e poi estesa alla copertura del fenomeno a livello globale (fig. 3).

Sulla cartina geografica sono resi visibili i casi dei contagi, indicati con un pallino rosso. Più grandi sono le dimensioni dei pallini e maggiore è la concentrazione delle vittime del contagio.

Non si tratta però di una visualizzazione statica, con la restituzione della globalità della situazione, ma di una visualizzazione interattiva, che in quanto tale consente al destinatario dell'informazione non soltanto di accedere all'immagine globale, sinottica e in continuo movimento, dell'estensione dell'epidemia ma di poter analizzare nel dettaglio, servendosi del puntatore del mouse e della funzione di zoom, un punto specifico sulla mappa.

In questo modo è possibile indagare, con un dettaglio che va dalla singola regione alla città, anche l'estensione dell'epidemia per numero di casi. In alto a sinistra, si trovano il numero di contagiati e l'ora dell'aggiornamento, rispetto al caricamento automatico dei dati dalle fonti..$^{10} \mathrm{~A}$ destra, invece, il numero dei morti e le persone ricoverate. In basso a destra un grafico con l'andamento del contagio su base giornaliera, che nell'immagine di fig. 3 ne evidenzia l'incremento.

Il problema di una visualizzazione come questa, a fine gennaio molto efficace nell'evidenziare le criticità sul circoscritto territorio della provincia di Wuhan, è risultato evidente con il passare dei mesi e l'incrementarsi esponenziale dei casi: paradossalmente, è stata la distribuzione capillare (e perfettamente aderente ai dati, come la celebre mappa di Borges al territorio) dei pallini rossi a rendere via via illeggibile il fenomeno.

8 Per esempio la VizGallery di Tableau Public sul COVID-19, creata a gennaio 2020 e aggiornata giornalmente, conta oltre 28.000 visualizzazioni di dati, tutte di libero accesso e catalogate secondo la tipologia dei dati che trattano (Governments, Healthcare \& Public Health, Economy \& Business).

9 Sull'espressione «flatten the curve» e sulle sue origjni (a febbraio 2020, in un articolo di Drew Harris per l'Economist) il New York Times ha dedicato un interessante articolo (cfr. Siobhan 2020).

10 La visualizzazione fornisce anche tutti i riferimenti alle fonti di dati su cui è basata, nello specifico i principali enti sanitari internazionali e nazionali tra cui l'Organizzazione Mondiale della Sanità (OMS/WHO); i Centers for Disease Control and Prevention (CDC) americani; l'European Centre for Disease Prevention and Control (ECDC); e altri come i CDC China; NHC e DXY. È costantemente aggiornata ed è consultabile all'indirizzo <https://coronavirus.jhu.edu/map.html>. 


\section{Dcula ${ }^{\text {Flux__aggi }}$ \\ Occhio semiotico sui media | Semiotic eye on media}

Valentina Manchia | Mappare il virus

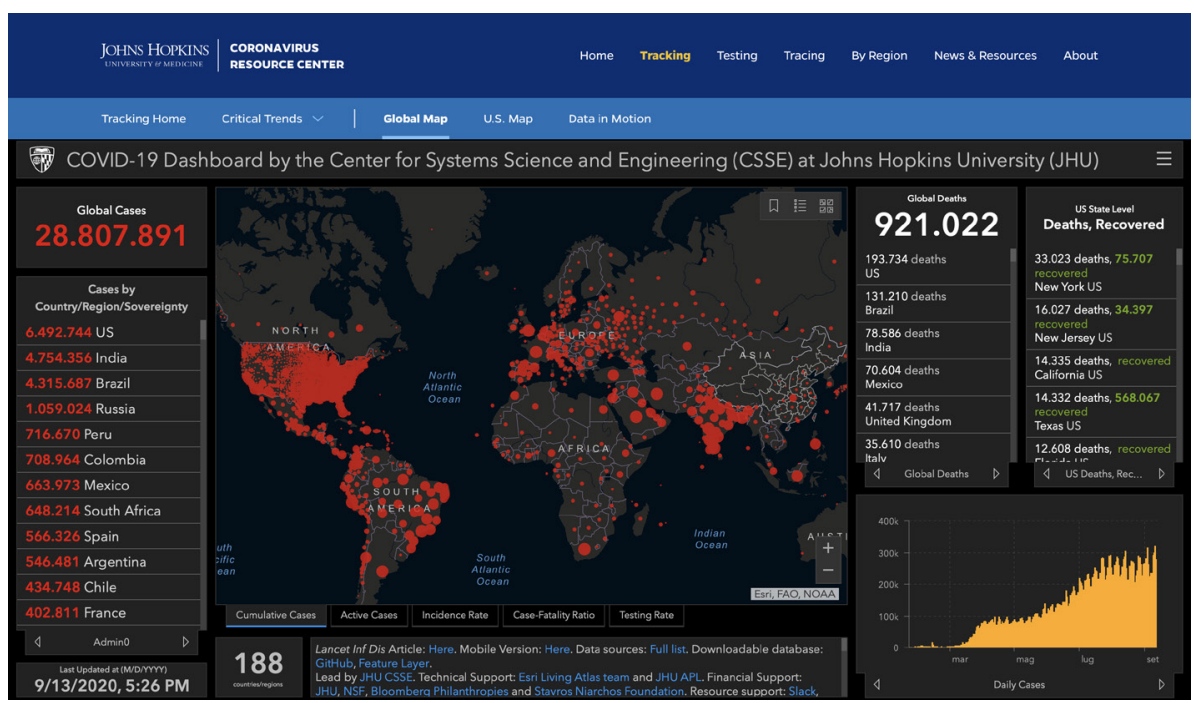

Figura 3. La dashboard complessiva della visualizzazione della Johns Hopkins University, al 13 settembre 2020.

Detto altrimenti, nel momento in cui il dato da rappresentare raggiunge proporzioni globali e allo stesso tempo numeri e dimensioni da big data, possiamo davvero essere certi di comprenderlo attraverso una visualizzazione che voglia offrircelo in tutta la sua totalità?

Di certo la dashboard della Johns Hopkins University, se utilissima per riassumere il costante incremento a livello mondiale dei casi di COVID-19, fornendo quasi un'ostensione diretta della della gran quantità di casi rilevati, individuati e rappresentati dettagliatamente,, non consente di operare agevolmente confronti tra i dati. Dal punto di vista dell'organizzazione dei dati, dunque sul piano del contenuto, ciò che è reso pertinente è soltanto la numerosità assoluta dei casi, che contrappone paesi più colpiti a paesi meno colpiti; dal punto di vista delle modalità espressive adottate, e dunque della messa in discorso, all'utente-osservatore è infatti richiesto di selezionare su un solo punto della mappa tra gli altri, concentrandosi dunque solo una zona per volta.

L'immagine che ne risulta, e che si è formata nel corso dei mesi proprio su questa dashboard, è di una proliferazione vertiginosa ed esponenziale di una presenza avversa, quella del virus, su un territorio prima incontaminato. Un'enorme macchia rossa in movimento che mostra ma non spiega, alla quale occorre affiancare altri approfondimenti, altre interpretazioni e altre immagini per tentare non solo una ripresa ravvicinata, esatta e puntuale, del virus ma un ritratto che ci aiuti a cogliere aspetti che da questa sorta di "presa diretta" finiscono per sfuggire.

Non è forse un caso che all'imporsi di un fenomeno globale e massivo (e, ricordiamolo, invisibile, e che quindi necessita di trovare una forma visiva per essere individuato e compreso) si sia optato anche per altre modalità di visualizzazione, capaci di restituirlo non solo a partire da dati quantitativi ma a partire da altre tipologie di dati. 
Valentina Manchia | Mappare il virus

\section{La narrazione dell'epidemia e altre forme di visualizzazione}

L'adozione di prospettive diverse da cui guardare ai dati, nel caso dell'epidemia di COVID-19, è stata da subito evidente, proprio per la necessità non solo di vedere tutto, di avere un accesso totale e capillare a tutti i dati accessibili, ma di vedere per capire, e per intervenire. ${ }^{11}$

Da più parti, infatti, e sin dai primi tempi del lockdown, è emersa l'esigenza di approfondire il fenomeno non solo attraverso la semplice ricezione dei dati ufficiali e l'invito a conformarsi ai comportamenti corretti per "abbassare la curva", ma anche attraverso altre "forme di dati", capaci di descrivere il fenomeno nella sua complessità. Il virus, infatti, com'è noto, non potrebbe prosperare né circolare senza entrare in simbiosi con l'uomo - ed è dunque sulle persone, sulle relazioni tra loro, e sulle comunità in cui vivono che occorre puntare l'attenzione se si vuole capire qualcosa di più del fenomeno.

Molte e diverse sono state le forme di visualizzazione adottate, da parte di testate giornalistiche o di team di data analysts, di semplici cittadini o blogger, e sarebbe impossibile passarle tutte in rassegna.

Ci sembra, però, di poter distinguere tra due categorie di operazioni alternative: le operazioni di visualizzazione che hanno adottato scelte altre dal punto di vista della raccolta dei dati, considerando come pertinenti dati e informazioni al di fuori delle fonti ufficiali; e le forme di visualizzazione che hanno invece messo in campo dei nuovi strumenti per restituire gli stessi dati, operando di fatto una messa in discorso radicalmente nuova dei dati ufficiali, con degli effetti di senso completamente inediti.

\subsection{Ripartire da nuovi dati. Dai big data ai thick data}

Nel primo orientamento rientrano tutte le operazioni di raccolta e di design dei dati che hanno deliberatamente scelto vie alternative a quelle dei dati ufficiali, opponendo a una posizione che pretende di essere esaustiva solo perché si rivolge ai grandi numeri uno sguardo più fine sul fenomeno - e allontanandosi dalla statistica classica per avvicinarsi piuttosto a un approccio etnografico ai dati.

L’idea portante dietro agli approcci alternativi è che dai dati ufficiali, che colgono l'andamento del virus solo quando si è di fatto convertito in un risultato (la vittima, o il contagiato) possa emergere soltanto un quadro parziale - una sorta di istantanea, ma del già stato, senza alcuna possibilità di attingere al come, e al perché, di quello che è accaduto e continua ad accadere. In risposta all'orientamento - che potremmo definire funzionalista - delle visualizzazioni ufficiali, diretto solo alla restituzione "trasparente" dei numeri così come sono, il nuovo orientamento propone infatti un'immersione nel fenomeno epidemico in quanto processo, e non soltanto come un risultato, proponendosi di visualizzare, di mettere in discorso in forma diagrammatica,

11 Siamo d'accordo con Thürlemann (2008) quando sostiene che ogni immagine diagrammatica è una machine à penser, una macchina capace di produrre delle performances cognitive (capire) o pragmatiche (trovare o fare) in chi le guarda (Thürlemann 2008: 137-150). 


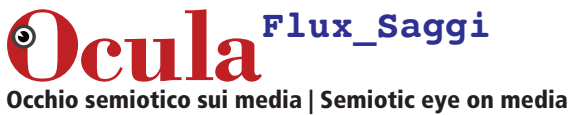

Valentina Manchia | Mappare il virus

quello che è il tessuto di azioni, relazioni, contatti ed emozioni tra le persone e che muove i loro comportamenti. ${ }^{12}$

Da qui l'idea di mettere a punto delle procedure per arrivare a "estrarre dei dati" da questo tessuto di azioni, comportamenti e relazioni, dati che proprio per la loro natura non sono più da considerarsi quantitativi, e basati statisticamente su grandi numeri, ma qualitativi. Il riferimento più frequente, in questo nuovo paradigma, è all'opposizione tra i big data e i cosiddetti thick data, dati "spessi" o "densi". Secondo la definizione che ne fornisce l'etnografa Tricia Wang, la prima a utilizzare questo termine:

Thick Data is data brought to light using qualitative, ethnographic research methods that uncover people's emotions, stories, and models of their world. It's the sticky stuff that's difficult to quantify. It comes to us in the form of a small sample size and in return we get an incredible depth of meanings and stories. Thick Data is the opposite of Big Data, which is quantitative data at a large scale that involves new technologies around capturing, storing, and analyzing. (Wang 2016)

I thick data sono quindi dati di un'altra natura: richiedono di essere "estratti” attraverso procedure etnografiche (quindi di osservazione e di ascolto) e allo stesso tempo di essere trattati come base per ottenere nuova conoscenza del fenomeno. Quello che ci sembra rilevante, qui, è proprio l'insistenza su un dato che è spesso, denso, pesante (tutt'altro che trasparente e immediato), che contiene allo stesso tempo le "storie" e le tracce delle "persone" cui quelle storie appartengono, e che necessita, per la sua stessa natura, di essere trasformato in discorso da qualcuno che ne assume, consapevolmente ed esplicitamente, la complessità.

Il riferimento di Wang, nel coniare l'espressione thick data, è a Clifford Geertz (1973) e alla «thick description» dell'antropologia e dell'etnografia, di cui in nessun caso le descrizioni possono intendersi come la perfetta restituzione di un "dato" oggettivo ma piuttosto come restituzioni di un fenomeno da uno specifico punto di vista, quello dell'analista. ${ }^{13}$

Potremmo però guardare, altrettanto utilmente, visto che qui ci occupiamo di immagini di dati, alla distinzione tra big data e thick data attraverso l'opposizione di Louis Marin tra trasparenza e opacità e da qui considerare, nello specifico, il modo in cui le strategie comunicative e retoriche legate all'introduzione dei thick data sulla scena della visualizzazione dei dati assumono fortemente, e come un valore, la propria presa di posizione sui dati. ${ }^{14}$

12 Da questo punto di vista, sarebbe interessante leggere il paradigma dei thick data (con la valorizzazione dell'attingere a nuove e più radicali fonti) come la messa in discorso di una scelta di testualizzazione radicalmente differente, nel senso di fissazione schematica di uno stadio intermedio di significazione. Il riferimento è alla definizione di testualizzazione come di «un arresto di questo percorso [del percorso generativo], a un momento qualsiasi del processo, e la sua deviazione verso la manifestazione» (Greimas, Courtés 1979-1986: 359).

13 Geertz (1973), tra l'altro, parla dell'approccio etnografico come di una «stratified hierarchy of meaningful structures».

14 Su trasparenza e opacità cfr. in particolare Marin (1994: 109-123 e 191-203, tr. 
Valentina Manchia | Mappare il virus

Lontani dal modello funzionalista cui gli analisti dei big data si richiamano, legato a una sedicente restituzione perfetta e trasparente dei dati, i nuovi analisti dei dati accettano e sottolineano il valore del proprio sguardo sui dati e dei metodi "caldi" (interviste, ricerche, documentazione, testimonianze) con cui questi dati vengono ottenuti.

Può essere dunque interessante, in una prospettiva semiotica, rendere conto del modo in cui le forme di restituzione di queste nuove tipologie di dati possano maggiormente rendere conto della connessione tra soggetti e azioni e delle loro ricadute successive. Un aspetto che, come abbiamo visto a proposito della pur breve disamina della comunicazione istituzionale italiana, non è indagato ma resta solo sottotraccia, dal momento che a essere esibita è l'evidenza dei risultati del virus, in termini di incidenza sotto forma di numero di vittime e di contagiati.

Un primo modo di descrivere l'impatto, a vari livelli, del fenomeno pandemico nella società attuale è stato quello di attingere alle storie e alle esperienze dei singoli durante il lockdown e trattarle come dati, a livello sistemico ma allo stesso tempo senza perdere il contatto con la singola, densa fonte individuale dei dati. Così per esempio nel progetto Design for Emergency, capitanato da Paolo Ciuccarelli alla Northeastern University di Boston e tuttora in corso, di cui sono disponibili le prime visualizzazioni dopo una campagna di raccolta dati sul territorio italiano, che è durata dal 15 al 29 marzo 2020 e ha raccolto 1748 risposte (fig. 4).

La resa finale è molto astratta, quasi asettica, e pensata per essere completamente intuitiva, basata com'è sull'utilizzo di forme circolari molto semplici che funzionano intuitivamente come i diagrammi di Eulero-Venn. Allo stesso tempo, però, è sufficiente interagire con la visualizzazione interattiva per notare il modo peculiare in cui i dati del sondaggio vengono posti sotto una nuova luce, quella di un "sentire comune" e dei valori condivisi (i grandi insiemi) all'interno del quale trovano posto i valori di ognuno, sia attraverso la restituzione del dato etnografico (le parole esatte alle domande del sondaggio) che attraverso la resa, dal punto di vista topologico, del gruppo inglobante come insieme di unità distinte ma non disperse.

Un modo per intrecciare i fili, isolati ma comunque paralleli, dei membri di una comunità coesa ma diffusa (quella italiana durante il lockdown, il periodo in cui è stata lanciata l’iniziativa e sono stati raccolti i dati).Un altro

it.). Qui proponiamo di schierare i big data dal lato della trasparenza e i thick data dal lato dell'opacità seguendo i discorsi e gli orientamenti di valore secondo cui i due paradigmi si costituiscono. Naturalmente, e si vedrà meglio più avanti, la nostra posizione è più complessa: seguendo Marin, infatti, trasparenza e opacità non sono mai mutualmente esclusive ma piuttosto occorre riconoscere che ogni immagine è trasparente, nel momento in cui dà accesso al suo oggetto, ma allo stesso tempo opaca, perché è impossibile che si dia una rappresentazione che non inglobi, insieme al suo oggetto, anche lo sguardo che la costituisce come immagine. Detto altrimenti, anche dietro l'artefatto visivo apparentemente più trasparente, volto a dirigere l'attenzione verso il solo oggetto rappresentato (nel nostro caso, a produrre un effetto di restituzione dei "puri" dati), resta un'irriducibile opacità, che è la traccia dell'intenzione (nel nostro caso, progettuale) che lo ha prodotto. Su trasparenza e opacità nei big data ci permettiamo di rimandare anche a Manchia (2015). 


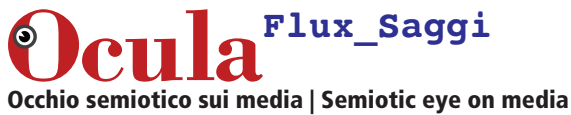

Valentina Manchia | Mappare il virus

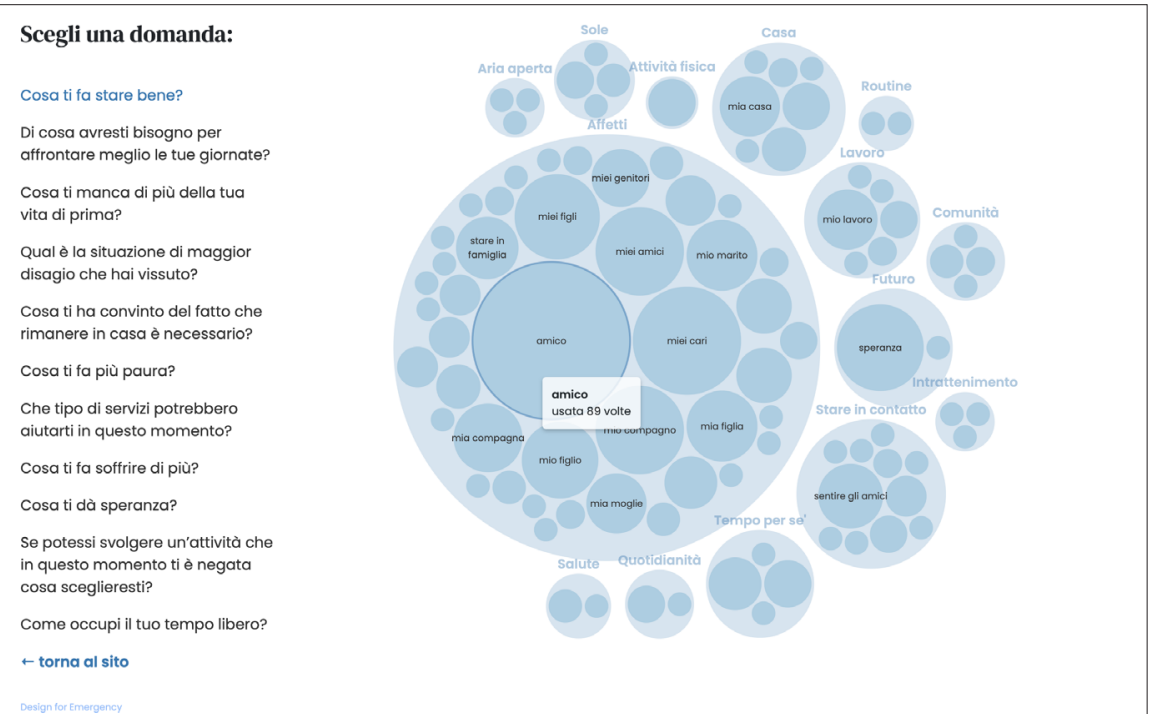

Figura 4. Screenshot dalla visualizzazione interattiva di Design for Emergency, di Sara Colombo, Paolo Ciuccarelli, Danilo Di Cuia, Piero Molino, Sara Perozzi, Sara Tonelli, Marco Guerini (CC BY 4.0).

esempio è quello del progetto Precision for Covid della Surgo Foundation, e in particolare la COVID-19 Community Vulnerability Index map che descrive la vulnerabilità del territorio americano di fronte alla pandemia e rende visibili ed evidenti, combinando indicatori specifici per COVID-19 con l'indice di vulnerabilità sociale (SVI) dei Centers for Disease Control and Prevention (CDC), i diversi modi in cui alcune comunità sono più vulnerabili di altre. La mappa interattiva utilizza la visualizzazione coropletica, ovvero le diverse aree sono colorate in modo da evidenziare gli indici statistici applicati, ma è possibile, con il puntatore, ottenere informazioni sullo specifico indice di vulnerabilità di ogni territorio.

Alla mappa coropletica interattiva, che fa da cornice, si aggiunge però un'ulteriore visualizzazione che consente di analizzare la vulnerabilità specifica di ogni territorio attraverso i parametri di cui è composta, mostrando la complessità di fattori (politici, sociali, economici) che contribuiscono al propagarsi del virus (fig. 5).

In questo modo l'incidenza del virus non è trattata come l'irruzione di un Anti-soggetto di segno negativo sulla scena, ma è intrecciata alle altre variabili di segno negativo già pendenti su un territorio.

Nella restituzione dei dati statistici della dashboard della Johns Hopkins University, infatti, l'opposizione fondante era quella tra uno standard e la deviazione dallo stardard, ovvero tra "sani" o aree "COVID-free" (la percentuale di popolazione al di fuori della statistiche governative, le zone grigie della mappa mondiale)", e "malati" o "aree COVID" (sotto gli incombenti pallini rossi), capaci di contaminare i "sani" (in modi anche laterali e imprevedibili, come per gli asintomatici): con il conseguente effetto, dal punto di vista della messa in discorso e della resa visiva generale, di un fenomeno dilagante e impossibile da maneggiare. 
Valentina Manchia | Mappare il virus

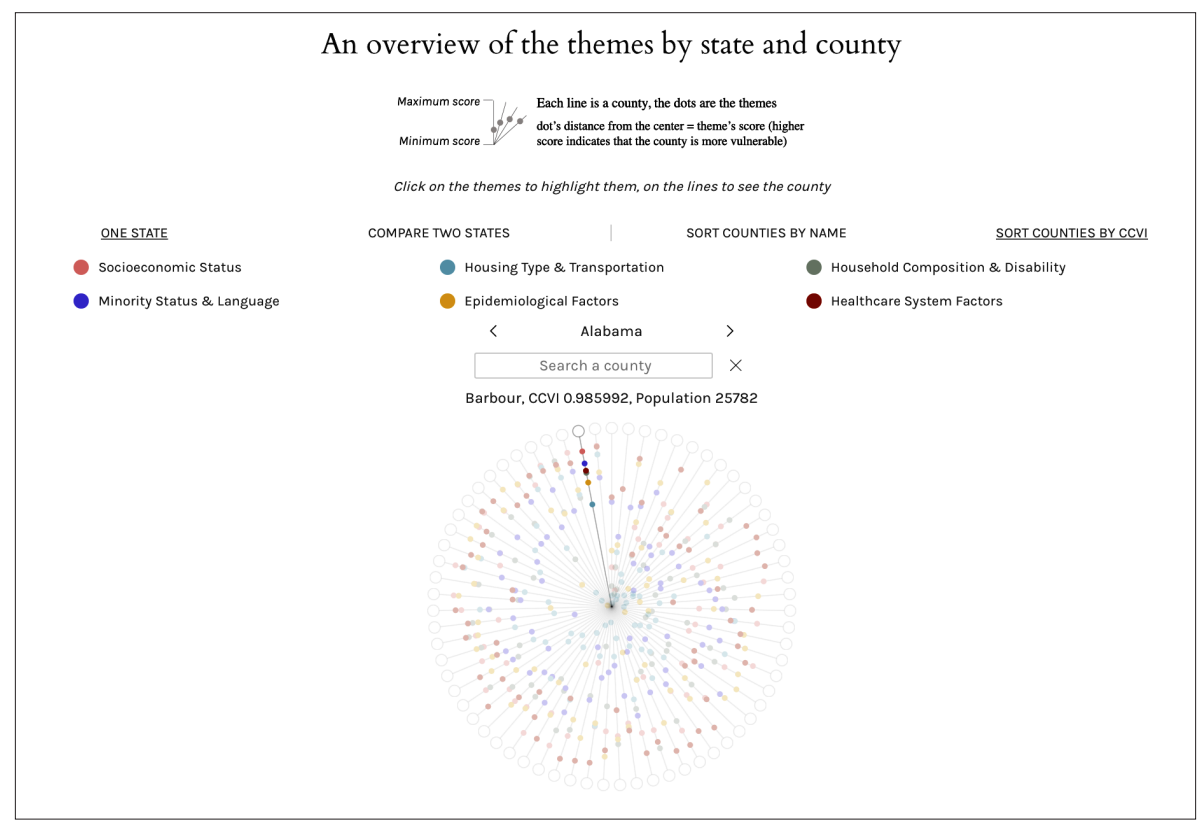

Figura 5. Screenshot da una delle visualizzazioni interattive di The COVID-19 Community Vulnerability Index (CCVI), di Precision for Covid, Surgo Foundation, design di Federica Fragapane, development di Paolo Corti.

Grazie all'operazione caldeggiata da Precision for Covid, invece, emerge un quadro molto più complesso, in cui il virus non ricopre la posizione dell'unico nemico da combattere ma è un fattore di rischio che può essere commisurato, valutato e magari controllato con gli opportuni interventi lungo le varie criticità locali. Le variabili tematiche che potrebbero intrecciarsi in modo critico al virus (lo status socioeconomico, le condizioni abitative, la presenza di eventuali disabilità nel nucleo familiare, l'appartenenza a una minoranza, i fattori epidemiologici, le strutture sanitarie) sono qui rappresentate da pallini di diversi colori, la cui posizione lungo ogni raggio indica il peso della variabile nel sistema regionale preso in considerazione (la forma circolare in cui si inscrivono i raggi, invece, è lo stato di riferimento).

\subsection{Guardare ai dati con altri occhi. L'approccio narrativo ai big data}

Un'altra strada che è stata percorsa per aggiungere maggiore complessità (e ulteriore spessore) alla descrizione del fenomeno è quella della messa a punto non di nuove tipologie di dati, ma di nuovi strumenti di interpretazione e di visualizzazione degli stessi dati. Ne è esempio il lavoro di Mona Chalabi, giornalista del Guardian, i cui lavori atipici di visualizzazione dei dati hanno avuto durante la pandemia grande risonanza su Instagram.

Un caso particolarmente interessante è legato alla cosiddetta questione razziale emersa, durante le settimane, a proposito della diffusione del virus.

È risultato infatti, dai dati ufficiali, che negli Stati Uniti i cittadini appartenenti a minoranze etniche sono stati colpiti in misura sproporzionata dall'epidemia di COVID-19 rispetto a quanto è accaduto per la popolazione bianca. 


\section{Ocula \\ Flux_Saggi \\ Occhio semiotico sui media | Semiotic eye on media}

Valentina Manchia | Mappare il virus

Tuttavia, è stato possibile rilevare questa correlazione soltanto a pandemia avanzata, quando i singoli stati e il governo federale hanno iniziato a rendere accessibili anche i dati disaggregati per appartenenza etnica: solo a partire dal mese di aprile, pertanto, è iniziata l'analisi dell'incidenza del virus sulle minoranze sul territorio americano.

Una correlazione così rilevante è diventata immediatamente oggetto di ricerche e di approfondimenti, oltre che di visualizzazioni dei dati. Molte di esse, come questa visualizzazione interattiva della Kaiser Family Foundation di cui proponiamo uno screenshot in fig. 6, hanno adottato la classica forma della mappa coropletica di dati con l'adozione di un gradiente cromatico per evidenziare l’incidenza del virus sulla categoria selezionata.

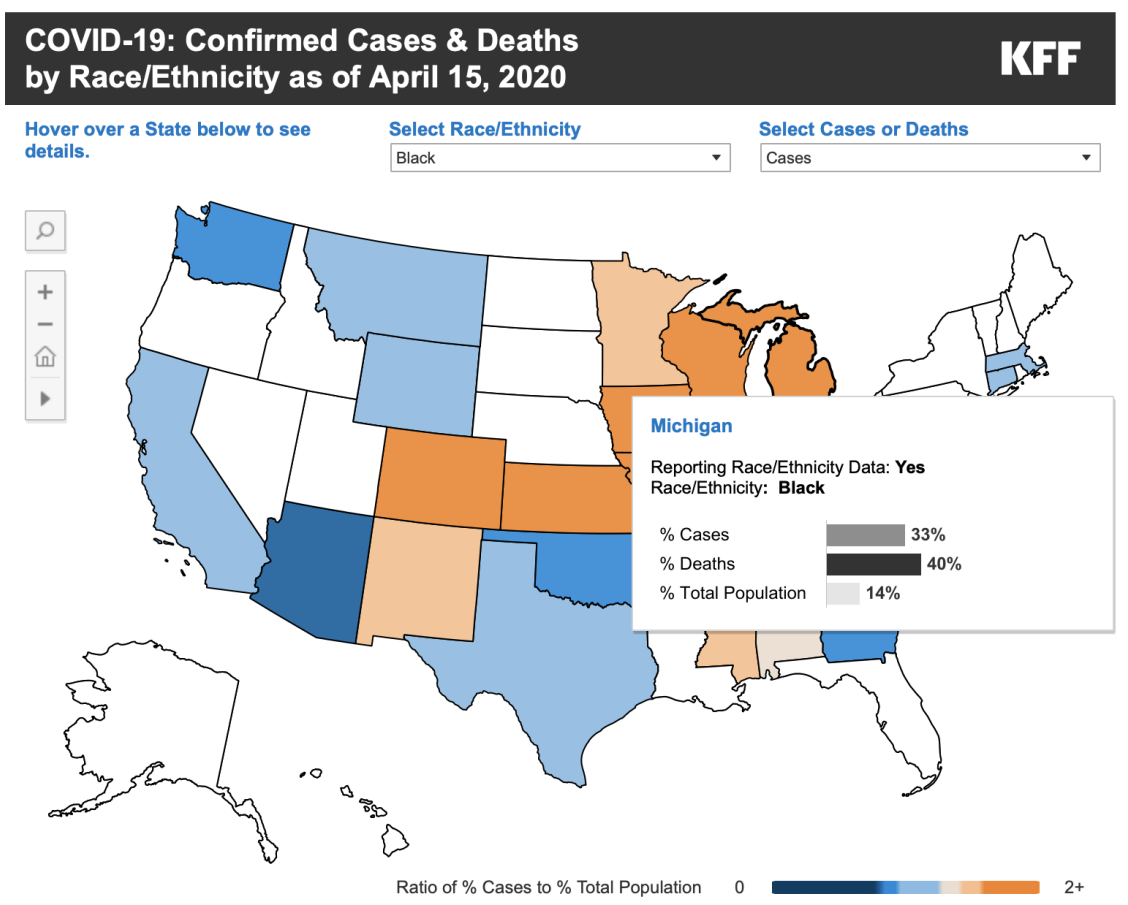

Figura 6. Screenshot dalla visualizzazione interattiva di Kaiser Family Foundation.

Come nel caso della dashboard della Johns Hopkins University, anche in questo caso è richiesto all'utente di farsi, per così dire, co-enunciatore con ogni sua singola scelta: qui, per esempio, selezionando la categoria Black e cliccando sullo stato del Michigan, si evidenzia un'incidenza molto alta del virus sulla popolazione nera - 40\% di vittime e 33\% di casi COVID-19 in un paese in cui più di un cittadino su dieci è nero ( $14 \%$ della popolazione totale), ma manca una visione di insieme che sia in grado di restituire un'immagine del fenomeno.

Quello che in ultima istanza la mappa racconta, infatti, è il peso delle minoranze (in termini di vittime e di casi conclamati) sull'economia generale di ogni stato, in cui la popolazione "di minoranza" è tale in opposizione a una 


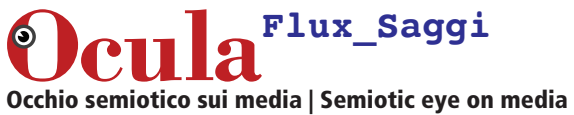

Valentina Manchia | Mappare il virus

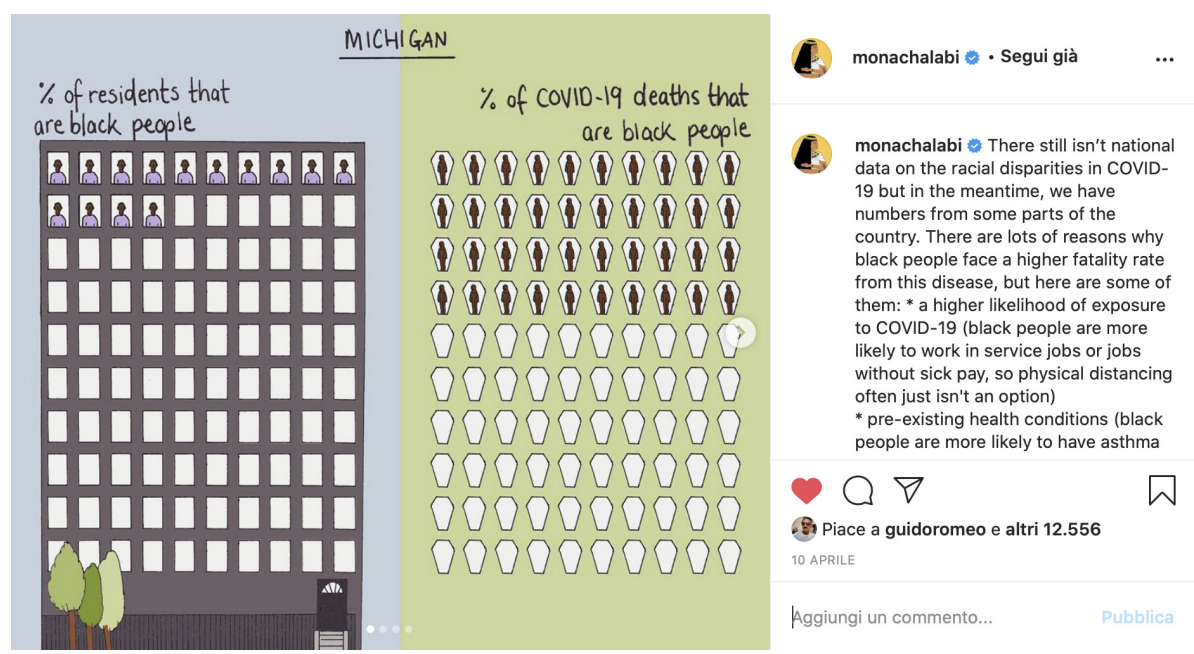

Figura 7. Una visualizzazione di Mona Chalabi pubblicata sul suo profilo Instagram, 10 aprile 2020.

popolazione "di maggioranza", totale. Con il pericoloso effetto secondario, in casi come quello del Michigan, in cui la popolazione nera è in una percentuale relativamente bassa (rispetto, per esempio, alla Louisiana, in cui più di un cittadino su 3 è nero) di sottostimare la situazione della minoranza, rispetto alla base di comparazione che è l'intera comunità del territorio nel suo complesso.

Va in tutt'altra direzione, invece, pur a partire dagli stessi dati la proposta visiva di Mona Chalabi (fig. 7), che come di consueto nei suoi lavori ibrida il linguaggio della visualizzazione dei dati con quello fortemente espressivo dell'illustrazione, risolutamente non convenzionale nell'ambito del data journalism.

Come si vede dalla data di pubblicazione del post (10 aprile 2020), i dati sono praticamente gli stessi forniti dalla visualizzazione della KFF, di poco successiva, basata su dati del 15 aprile 2020. Tuttavia, la messa in discorso dei dati a opera della data journalist del Guardian è molto differente, e per più di un motivo.

A livello del contenuto, innanzitutto, non emerge soltanto il doppio dato ascrivibile alla minoranza (il 14\% di «black people» tra gli abitanti e il 40\% di «black people» tra le vittime) ma una doppia contrapposizione: quella tra la totalità dei residenti e i cittadini neri, a sinistra (ogni finestra del condominio corrisponde a un punto percentuale, e così ogni bara nel cinitero) e tra le vittime afroamericane rispetto all'intera popolazione nera del Michigan.

La rappresentazione visiva e figurativa, infatti, non solo delle singole quantità in percentuale ma del contesto stesso in cui le quantità si situano (il condominio, a simboleggiare la comunità viva e attiva; e la distesa di bare, per i morti di COVID-19), ottenuta trasponendo prima la totalità percentuale in una matrice vuota (le finestre del condominio; le bare vuote del cimitero) e poi marcando le quantità, ovvero riempiendo le caselle della doppia matrice (le finestre e le bare), produce immediatamente questa visibile e tangibile doppia differenza, e dipinge immediatamente un quadro molto più complesso: quello 


\section{Ocula \\ Occhio semiotico sui media | Semiotic eye on media}

Valentina Manchia | Mappare il virus

di un territorio in cui i neri sono solo una minima parte, una piccola comunità nella comunità che tuttavia ha subito grandi perdite umane.

Ad accompagnare l'illustrazione una lunga didascalia che agisce da cornice e da ancoraggio referenziale, riconducendo la grande incidenza del virus sulle comunità nera alle peggiori condizioni di vita di cui la minoranza soffre:

There still isn't national data on the racial disparities in COVID-19 but in the meantime, we have numbers from some parts of the country. There are lots of reasons why black people face a higher fatality rate from this disease, but here are some of them: * a higher likelihood of exposure to COVID-19 (black people are more likely to work in service jobs or jobs without sick pay, so physical distancing often just isn't an option)

* pre-existing health conditions (black people are more likely to have asthma and diabetes which weaken the immune system - those conditions are linked to a person's environment, see my previous illustrations on racial disparities in air pollution and access to fresh, affordable food)

* racism within the healthcare system that means that black people receive poorer quality care when they do get sick.

Nell'impatto di una trasposizione visiva di questo tipo conta molto la scelta di puntare, dal punto di vista del contenuto, sulla doppia opposizione con un solo termine marcato da entrambe le parti - doppia opposizione che un mezzo figurativo come l'illustrazione non può mancare di mostrare. Ben diverso sarebbe stato il risultato, infatti, se al posto dell'uso volutamente naïf e lo-fi dell'illustrazione l'autrice avesse utilizzato altre forme più astratte di visualizzazione.

È poi anche la scelta di modulare la matrice astratta (le 100 unità della base percentuale) in forma visiva a garantire l'immediatezza della lettura. Nella resa espressiva e immediata della doppia opposizione (tra le due metà dell'illustrazione e, in ogni sezione, tra matrice vuota e quantità marcate), infine, assume un ruolo importante anche la figurativizzazione della matrice da due diversi punti di vista nettamente opposti: la visione frontale del grande condominio-Michigan, cui si affacciano figure stilizzate, e la visione dall'alto della distesa di bare.

In uno scenario mediatico in cui la visualizzazione dei dati assume sempre più un ruolo di primaria importanza, e in cui le forme classiche di visualizzazione (diagrammi, grafici, istogrammi) iniziano ad assumere un valore concreto anche per i fruitori più ingenui e meno accorti di tali contenuti (si veda quanto detto prima su «flatten the curve»), Mona Chalabi usa consapevolmente l'illustrazione come uno strumento espressivo capace sì di restituire i dati ma allo stesso tempo di aggiungere alla trasposizione delle statistiche quel punto di vista che i teorici dei thick data contro i big data cercano di recuperare. Una buona dimostrazione che non è solamente nel tipo di dati o di informazioni che si maneggiano - nei due ultimi casi, come si è visto, erano in gioco le stesse percentuali - che sta la differenza tra big e thick data.

Piuttosto, ci sembra, è sempre questione di diverse strategie di messa in discorso. E questo sia dal punto di vista di quello che viene reso pertinente sul piano del contenuto (abbiamo infatti cercato di mostrare come anche su- 


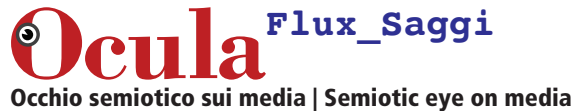

Valentina Manchia | Mappare il virus

gli stessi dati si possa gettare una luce molto diversa) sia a partire dai mezzi espressivi utilizzati (la freddezza scientifica - che rischia di essere presa per invisibile, anche se ovviamente non lo è - delle forme diagrammatiche classiche vs. il linguaggio personale e "caldo" dell'illustrazione).

In ogni caso, ogni visualizzazione di dati - proprio come ogni altro testo significa all'interno delle specifiche strategie comunicative da cui dipende, ed esibisce tracce che possiamo interpretare alla luce di una regia molto precisa del discorso (di una specifica istanza dell'enunciazione, capace di stringere in modo coerente tutte le operazioni effettuate sui dati e sulle loro relazioni). ${ }^{15}$

Ci sembra dunque interessante, al di là e oltre la contrapposizione - troppo semplice, tra big data e thick data, continuare a osservare la superficie (ben profonda) di quello che possiamo chiamare discorso dei dati, aprendo a un'analisi delle strutture narrative e discorsive al lavoro in questi artefatti comunicativi.

\section{Bibliografia}

Aït-Touati, Frédérique

2020 «Nous ne sommes pas le nombre que nous croyions être» relire Les Microbes de Bruno Latour, <https://aoc.media/ critique/2020/o8/18/nous-ne-sommes-pas-le-nombre-que-nous-croyionsetre-relire-les-microbes-de-bruno-latour> “AOC”, 23 mars 2020.

Bastide, Françoise

1985a "Essai d'épistemologie à partir d'un texte technique sans prétention: une invention peu connue des frères Lumière", Fundamenta scientiae, VI, 2, pp. 127-150 (tr. it. "Saggio di epistemologia nato da un testo senza pretese", in Una notte con Saturno. Scritti semiotici sul discorso scientifico, a cura di B. Latour, P. Fabbri, Roma, Meltemi, 2001, pp. 215-250).

1985b "Iconographie des textes scientifiques: principes d'analyse", Culture Technique, 14 (tr. it. "Iconografia dei testi scientifici. Principi d'analisi", in Una notte con Saturno. Scritti semiotici sul discorso scientifico, a cura di B. Latour, P. Fabbri, Roma, Meltemi, 2001, pp. 167-213).

2001 Una notte con Saturno. Scritti semiotici sul discorso scientifico, a cura di B. Latour, P. Fabbri, Roma, Meltemi.

Bonini, Tiziano

2020 "Making Sense of the Pandemic through Data: The Italian Case", <https:// data-activism.net/2020/06/bigdatasur-covid-making-sense-of-the-pandemicthrough-data-the-italian-case/>, 24 giugno 2020.

Cairo, Alberto

2013 The functional art. An introduction to information graphics and visualization, Berkeley, New Riders (tr. it. L'arte funzionale. Infografica e visualizzazione delle informazioni, Milano, Rizzoli, 2013).

15 Sull'istanza dell'enunciazione, seguendo Calabrese (1985), come specifica «messa in prospettiva» di un sistema dato, anche attraverso diverse forme discorsive, cfr. Lancioni (2013), ma anche Corrain-Lancioni (2012). 


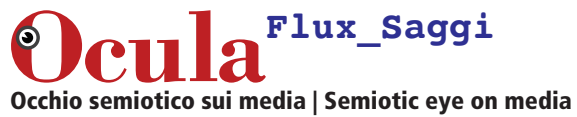

Valentina Manchia | Mappare il virus

Calabrese, Omar

1985 La macchina della pittura, Roma-Bari, Laterza (nuova ed. La macchina della pittura. Pratiche teoriche della rappresentazione figurativa tra Rinascimento e Barocco, Firenze, La Casa Usher, 2012).

Corrain, Lucia; Lancioni, Tarcisio

2012 "Geometrie del senso. Ripensare la semiotica dell'immagine a partire da $\mathrm{La}$ macchina della pittura di Omar Calabrese", E|C, pp. 1-12 <http://www.ec-aiss. it/includes/tng/pub/tNG_download4.php?record $\mathrm{ID}=657 \& \mathrm{KT} \_$download1 $=\mathrm{a} 6512 \mathrm{c} 6693 \mathrm{a} 3506026073 \mathrm{~b} 273 \mathrm{a} 6 \mathrm{ec} 755>$

Fabbri, Paolo

2001 Introduzione a Una notte con Saturno. Scritti semiotici sul discorso scientifico, a cura di B. Latour, P. Fabbri, Roma, Meltemi, 2001, pp. 9-23.

Geertz, Clifford

1973 "Thick Description: Toward an Interpretive Theory of Culture", in Id., The Interpretation of Cultures: Selected Essays, New York, Basic Books, pp. 3-30.

Goody, Jack

1977 The Domestication of the Savage Mind, Cambridge, Cambridge University Press (tr. it. L'addomesticamento del pensiero selvaggio, Milano, FrancoAngeli, 1981).

Greimas, Algirdas J., Courtés, Jacques

1979-1986 Sémiotique. Dictionnaire raisonné de la théorie du langage, I-II, Paris, Hachette (tr. it. Semiotica. Dizionario ragionato della teoria del linguaggio, Usher, Firenze, 1986, ora anche tr. parz. in Semiotica. Dizionario ragionato della teoria del linguaggio, Milano, Bruno Mondadori, 2007).

Lancioni, Tarcisio

2013 "Immagini in prospettiva. Forme e figure dell'enunciazione visiva", in Semiotica delle soggettività. Per Omar, a cura di M. Leone, I. Pezzini, Aracne, Roma, 2013, pp. 281-288.

Latour, Bruno

1984 Les Microbes: guerre et paix, suivi de Irréductions, Paris, Metailie.

1987 Science in Action. How to Follow Scientists and Engineers through Society, Harvard University Press (tr. it. La scienza in azione. Introduzione alla sociologia della scienza, Torino, Edizioni di Comunità, 1998).

1996 Petite réflexion sur le culte moderne des dieux faitiches, Le Plessis-Robinson, Synthélabo (tr. it: Il culto moderno dei fatticci, Roma, Meltemi, 2005).

2011 "La semiotica dei testi scientifici dopo il lavoro di Françoise Bastide", $E \mid C$, pp. 1-7. <http://www. ec-aiss.it/includes/tng/pub/tNG_download4. php?recordID=561\&KT_download1=2e2c930b441fad8 b874f7ba9a488f105>.

Latour, Bruno; Fabbri, Paolo

1977 "La rhétorique de la science. Pouvoir et devoir dans un article de science exacte", Actes de la Recherche en sciences sociales, Paris, Minuit (tr. it. in Semiotica in nuce I. I fondamenti e l'epistemologia strutturale, a cura di P. Fabbri e G. Marrone, Roma, Meltemi, 1999, pp. 260-279).

Latour, Bruno; Woolgar, Steve

1979 Laboratory Life. The Construction of Scientific Facts, Los Angeles, Sage Publications (ed. rivista, Laboratory Life: the Construction of Scientific Facts, Princeton, Princeton University Press, 1986. 


\section{Ocula ${ }^{\text {Flux_saggi }}$ \\ Occhio semiotico sui media | Semiotic eye on media}

Valentina Manchia | Mappare il virus

Manchia, Valentina

2015 "Gli Afghan War Logs di WikiLeaks riletti dal Guardian", in O. Gomez, M. Serra (eds.), Transparencia y secreto, atti del II Congresso internazionale GESC, Madrid, 20-22 novembre 2013, Madrid, Visor Libros, pp. 33-46.

Marin, Louis

1994 De la représentation, Paris, Le Seuil/Gallimard (tr. it. Della rappresentazione, a cura di L. Corrain, Milano, Mimesis, 2014).

Montanari, Federico (a cura di)

2020 "Epide(se)miologie e contagi. Dialogo con Paolo Fabbri", Ocula, pp. 1-10, $<$ https://www.ocula.it/files/OCULA-Documenti-MONTANARI-Ocula-docepidesemiologie_e_contagi.pdf>, 3 aprile 2020

Ong, Walter

1982 Orality and Literacy. The Technologizing of the word, London-New York, Methuen (tr. it. Oralità e scrittura. Le tecnologie della parola, Bologna, Il Mulino, 1986).

Roberts, Siobhan

2020 "Flattening the Coronavirus Curve", The New York Times, <https://www. nytimes.com/article/flatten-curve-coronavirus.html>, 27 marzo 2020.

Thürlemann, Félix

2008 “Diagramme ou regard diagrammatique?", Visible. Diagrammes, cartes, schémas graphiques, 4.

Wang, Tricia

2016 "Why Big Data Needs Thick Data", Etnography Matters, <https://medium. com/ethnography-matters/why-big-data-needs-thick-data-b4b3e75e3d7\#. xhtmw64pe>, 20 gennaio 2016.

Valentina Manchia Dottore di ricerca in Semiotica e Comunicazione simbolica presso l'Università di Siena, attualmente insegna al Politecnico di Milano, all'Alma Mater Studiorum - Università di Bologna, a ISIA Urbino e IAAD (Bologna e Torino), dove si occupa di semiotica, comunicazione e progettazione visiva, design e cultura visuale. Ha curato, per La Casa Usher, il numero di Carte Semiotiche dal titolo Immagini che fanno segno. Modi e pratiche di rappresentazione diagrammatica nelle informational images (2015). Suoi saggi e articoli sono apparsi, oltre che in pubblicazioni collegate a convegni nazionali e internazionali, su Lexia, E/C, Progetto grafico, Alfabeta2, Doppiozero. È membro del CROSS - Centro di Ricerca "Omar Calabrese" di Semiotica e Scienze dell'immagine. 